\title{
Deviation correction method for close-range photometric stereo with nonuniform illumination
}

\author{
Hao Fan, ${ }^{a, \dagger}$ Lin Qi, ${ }^{a, \dagger}$ Nan Wang, ${ }^{a}$ Junyu Dong, ${ }^{a, \star}$ Yijun Chen, ${ }^{a}$ and Hui $\mathbf{Y u}^{\mathrm{b}}$ \\ ${ }^{a}$ Ocean University of China, College of Information Science and Engineering, Qingdao, China \\ bUniversity of Portsmouth, School of Creative Technologies, Portsmouth, United Kingdom
}

\begin{abstract}
Classical photometric stereo requires uniform collimated light, but point light sources are usually employed in practical setups. This introduces errors to the recovered surface shape. We found that when the light sources are evenly placed around the object with the same slant angle, the main component of the errors is the low-frequency deformation, which can be approximately described by a quadratic function. We proposed a postprocessing method to correct the deviation caused by the nonuniform illumination. The method refines the surface shape with prior information from calibration using a flat plane or the object itself. And we further introduce an optimization scheme to improve the reconstruction accuracy when the three-dimensional information of some locations is available. Experiments were conducted using surfaces captured with our device and those from a public dataset. The results demonstrate the effectiveness of the proposed approach. $\odot 2017$ Society of Photo-Optical Instrumentation Engineers (SPIE) [DOI: 10.1117/1.OE.56.10.103102]
\end{abstract}

Keywords: photometric stereo; nonuniform illumination; deviation correction.

Paper 170682 received May 8, 2017; accepted for publication Sep. 13, 2017; published online Oct. 6, 2017.

\section{Introduction}

Photometric stereo is a well-established three-dimensional (3-D) reconstruction technique that uses images of a scene taken from the same viewpoint but under different illumination directions. ${ }^{1}$ Photometric stereo recovers gradients (or normal) of each surface facet from captured images, and then the surface height can be integrated from the gradient field. ${ }^{2-4}$ Compared with conventional geometric techniques, such as binocular stereo ${ }^{5}$ and structure from motion, ${ }^{6}$ photometric stereo produces better surface details with simpler calculation. $^{7,8}$

Classical photometric stereo requires uniform and collimated illumination. ${ }^{9}$ However, the light sources employed in practical photometric stereo setups are usually nonuniform, such as point light sources. The point light sources are placed far away from the object to approximate ideal illumination conditions. ${ }^{10,11}$ But this solution cannot be used in practical applications that provide limited device space, such as those used for underwater robots ${ }^{9,12}$ and monocular laparoscopy. ${ }^{13}$ In this situation, the reconstruction errors caused by the nonuniform lighting cannot be ignored. ${ }^{14,15}$ Figures 1(a) and 1(c) show the erroneous surface reconstruction result produced by classical photometric stereo under close-range light sources.

In this paper, we propose a postprocessing method to correct the surface shape recovered by using classical photometric stereo under nonuniform lighting. The method was inspired by our finding that when the light sources are evenly distributed around the object with the same slant angle, the classic photometric stereo introduces linear deviations to the recovered surface gradients. The surface height, which can be integrated from gradients, exhibits a quadratic noise. We, therefore, propose to correct the deviation in the gradient field.

\footnotetext{
*Address all correspondence to: Junyu Dong, E-mail: dongjunyu@ @ouc.edu.cn
}

†These authors contributed equally to this work.
Depending on the available prior knowledge, our proposed method consists of three strategies for different cases. When the lighting calibration or 3-D information of any known surface points is not given, the gradients can be corrected by simply removing the fitted low-frequency deviation, which is denoted by "gradient self-correction (GSC)." When the 3-D information of some surface points is available, e.g., from laser triangulation or binocular stereo, we propose an optimization algorithm to correct the surface shape, which is denoted by "optimized gradient self-correction (OGSC)." The above two methods can well handle those surfaces whose global normal is pointing to the camera. Otherwise, the lighting calibration will be required, which is denoted by "optimized gradient calibration (OGC)." The experiments show that our approach is more accurate than traditional methods under close-range lighting.

The paper is organized as follows. We review the related work and the basic photometric stereo theory in Sec. 2. Analysis of the gradient deviation in classic photometric stereo is presented in Sec. 3, which is followed by the preliminary correction method. The optimization method is described in Sec. 5. In Sec. 6, we discuss the performance of the proposed methods. Finally, we conclude our work in Sec. 7.

\section{Background}

This section presents related work in correcting the normal and height deviation produced by photometric stereo under point light sources. A brief overview of the classic photometric stereo theory and the limitation when using point light sources are also presented.

\subsection{Related Work}

Photometric stereo was proposed by Woodham ${ }^{1}$ nearly 40 years ago. There are three fundamental assumptions to photometric stereo in the original approach: 

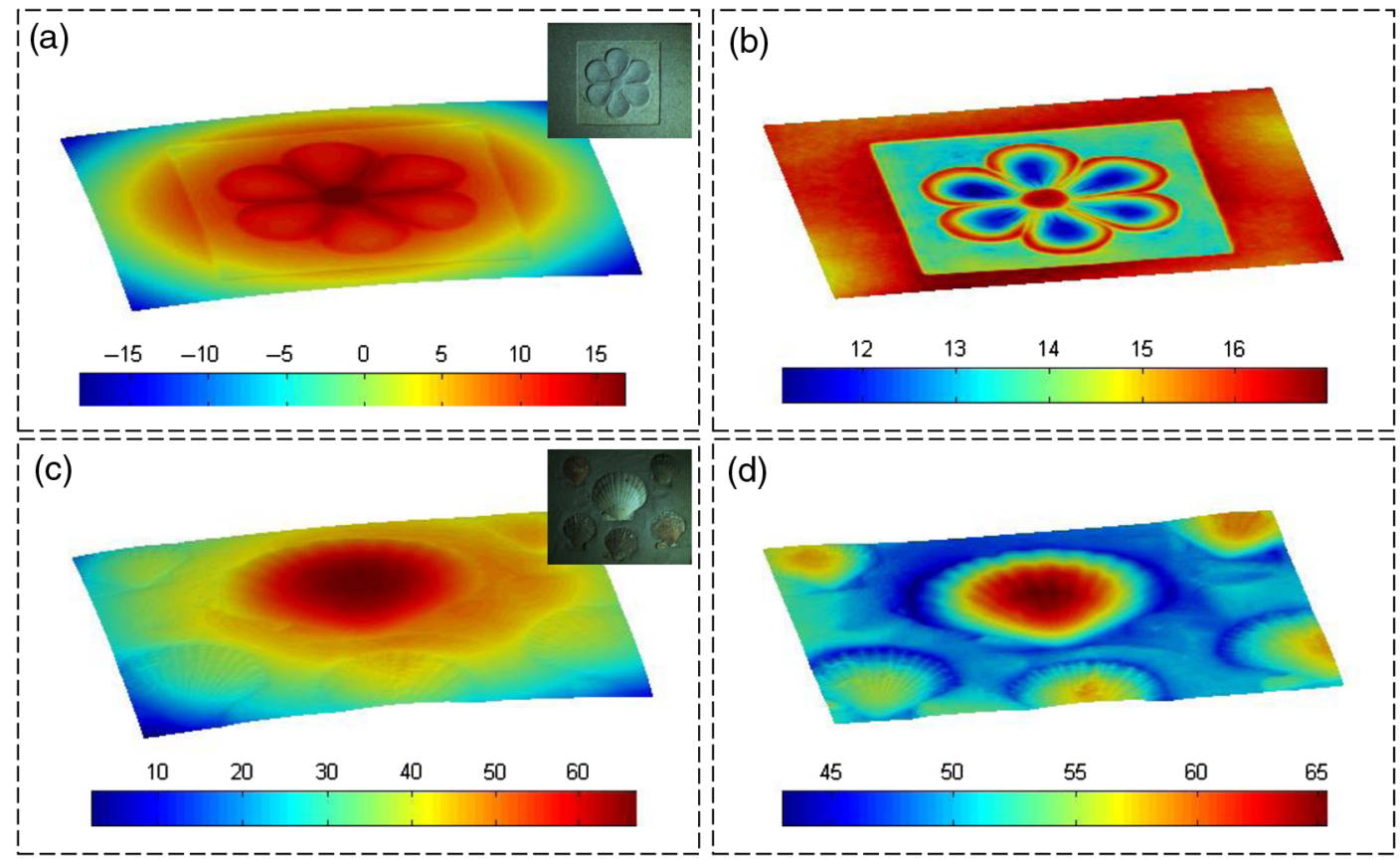

Fig. 1 (a) and (c) Surface reconstruction of a flat bas-relief slate and a flat flagstone with embedded shells using classical photometric stereo. (b) and (d) Corrected reconstruction results of the same objects using our proposed method.

- the surface is Lambertian,

- the light sources are placed at infinity for uniform collimated illumination, and

- the camera optics is equivalent to an orthographic projection.

However, these assumptions are not always met in some practical situations, which bring errors in the recovered surface shape. ${ }^{16}$ In this paper, we focus on the inaccuracies in the low-frequency components of the reconstructed shape introduced by close-range lighting. It is usually solved in two ways: modeling the direction and attenuation of illumination at each surface point or correcting the shape deviation afterward.

To remove the effect of nonuniform illumination, Angelopoulou and Petrou ${ }^{10}$ proposed the flat-fielding method (flat fielding), in which the light distribution of each light source is calibrated with a flat plane. The captured images were then corrected by dividing the calibration image. However, this method does not work for objects with different albedo. Light attenuation models were introduced to improve the accuracy. For point light sources, intensity was mostly computed with the inverse-square law. ${ }^{9,17-19}$ Nagesh et al. used point light sources in an underwater photometric stereo and iteratively solved light attenuation model parameters. Higo et al. attached a point light source to a handheld camera to add a photometric constraint to the multiview stereo problem. These works only modeled the inversesquared light attenuation, whereas Collins and Bartoli ${ }^{13}$ considered both distance and angular attenuations. Their model turns close-range photometric stereo to a nonlinear problem. Wetzler et al. ${ }^{20}$ and Logothetis et al. ${ }^{21}$ proposed using partial differential equations for close-range photometric stereo. Since the method can only handle two images due to computation complexity, researchers further proposed variational methods. ${ }^{22-24}$ Using a complex light, attenuation model can better explain the imaging process but introduces difficulties to calibration and computation.

Some approaches directly correct the height deviation produced by classic photometric stereo. Combining photometric stereo with geometric methods, such as binocular stereo and structured light, can significantly improve the accuracy. ${ }^{25-27}$ Horovitz and Kiryati ${ }^{14}$ proposed using sparse control points with known 3-D locations to correct the height deviation, and the performance depends on the key points selected.

The deviation caused by classic photometric stereo under point light sources is low-frequency noise. In this paper, we propose to remove the deviation using as few requirements as possible for the low cost and more practical purpose.

\subsection{Classic Photometric Stereo}

In classic photometric stereo setup, the $x y$-plane of the world coordinate system coincides with the image plane, whereas the $z$-axis is lined up with the optical axis of the imaging system. A surface point with coordinates $(x, y, z)$ maps onto the image point $(u, v)$. The height at the point is defined as $z=f(x, y)$, and the gradient components are defined as $p=\partial f(x, y) / \partial x$ and $q=\partial f(x, y) / \partial y$. If $\mathbf{n}$ denotes the surface normal vector, the unit surface normal is given as

$\hat{\mathbf{n}}=\frac{\mathbf{n}}{|\mathbf{n}|}=\frac{(-p,-q, 1)}{\sqrt{1+p^{2}+q^{2}}}$

The measured intensity $e_{k}$ for a single pixel under the light source $k(k \in[1, K])$ satisfies

$e_{k}=\rho\left(\hat{\mathbf{n}} \cdot \mathbf{l}_{k}\right)$,

where $\rho$ denotes the reflectance albedo of the point and $\mathbf{l}_{k}$ is the unit light direction of the $k$ 'th light source. 
By joining the $k$ measurements for the same pixel together, we have

$$
\mathbf{E}=\rho \hat{\mathbf{n}} \mathbf{L},
$$

where the $k^{\prime}$ th column of the matrix $\mathbf{E}\left(\mathbf{E} \in \mathbb{R}^{1 \times K}\right)$ represents the image intensity under the $k^{\prime}$ th light source and the $k^{\prime}$ th column of $\mathbf{L}\left(\mathbf{L} \in \mathbb{R}^{3 \times K}\right)$ is the $k^{\prime}$ th illumination direction $\mathbf{I}_{k}$.

If the columns of $\mathbf{L}$ are noncoplanar, $\mathbf{L}$ is nonsingular and invertible. So we can have

$\mathbf{W}=\rho \hat{\mathbf{n}}=\mathbf{E L}^{\dagger}$,

where $\mathbf{W}=\left(w_{1}, w_{2}, w_{3}\right)^{T}$ and $\mathbf{L}^{\dagger}$ denotes the pseudoinverse of $\mathbf{L}$. A minimum of $K=3$ is required for solving Eq. (4). Then the surface gradient components can be written as

$p=-\frac{w_{1}}{w_{3}}, q=-\frac{w_{2}}{w_{3}}$.

The gradients can be used to compute normal for each pixel using Eq. (1).

\subsection{Photometric Stereo under Point Light Sources}

In this paper, we discuss the situation that photometric stereo is implemented under close-range point light sources, which means the illumination is not uniform or collimated. The perspective projection and light attenuations should be considered. ${ }^{13}$

As shown in Fig. 2, the $x y$-plane is parallel with the image plane, and the $z$-axis is aligned with the optical axis of the imaging system. A surface point $A$ with coordinate $(x, y, z)$ maps onto the image point $A^{\prime}(u, v)$ under the perspective projection.

\subsubsection{Light direction}

If the coordinate of the $k^{\prime}$ th light source is $\left(l x_{k}, l y_{k}, l z_{k}\right)$, the light direction $\mathbf{I}_{k}(x, y)$ for a surface location $(x, y)$ can be written as

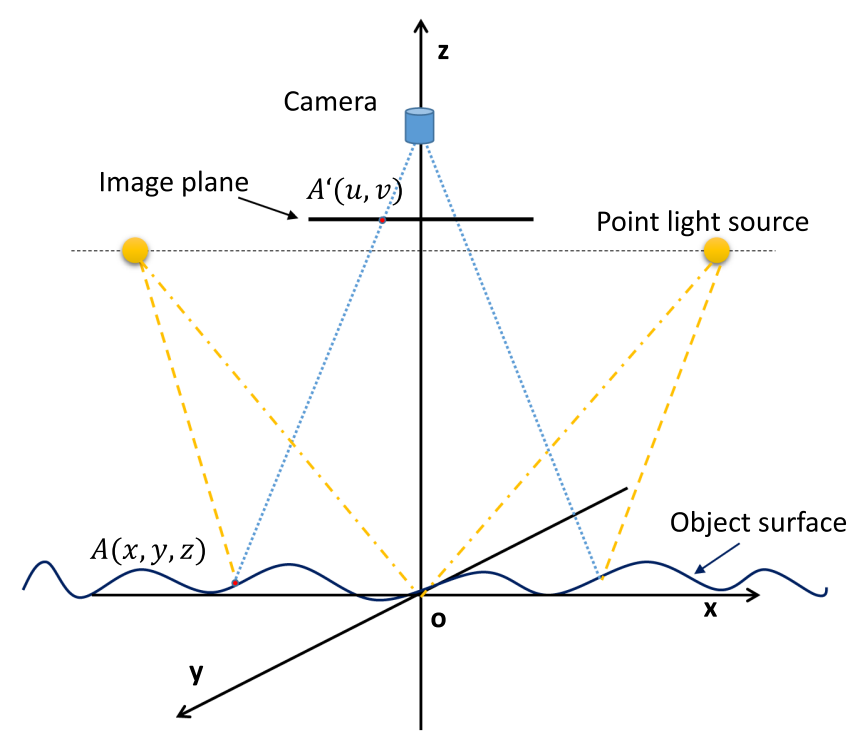

Fig. 2 Photometric stereo under point light sources. $\mathbf{l}_{k}(x, y)=\frac{\left(l x_{k}-x, l y_{k}-y, l z_{k}-z\right)}{\sqrt{\left(l x_{k}-x\right)^{2}+\left(l y_{k}-y\right)^{2}+\left(l z_{k}-z\right)^{2}}}$.

In this model, the light directions depend not only on $(x, y)$ but also on $z$.

\subsubsection{Light attenuation}

Two types of light attenuation are taken into consideration when point light sources are used. First, the light energy attenuates due to distance following the inverse-square law. Second, the light encounters radial attenuation, which means that the light is bright along a principal direction $\mathbf{v}_{k}$ and becomes less bright at angles further from the main direction. The light attenuation can be expressed by the multiplication of two factors ${ }^{20}$

$a_{k}(u, v)=\frac{\left[\mathbf{l}_{k}(u, v) \cdot \mathbf{v}_{k}\right]^{\mu}}{\left(l x_{k}-x\right)^{2}+\left(l y_{k}-y\right)^{2}+\left(l z_{k}-z\right)^{2}}$,

where $\mu$ is a radial attenuation coefficient.

The varying light direction and attenuation introduce nonlinear errors to the reconstructed surface normal, which is what we aim to correct in the following.

\section{Gradient Deviation in Classic Photometric Stereo}

We built up a photometric stereo device to capture pictures of objects as shown in Fig. 3. The light sources are evenly distributed around the objects with the same slant angle. The captured surface is globally flat and lies in $x y$-plane. The main direction of the point light source can be defined by a slant angle and a tilt angle. ${ }^{28}$ Different objects were photographed under varying tilt angles. More details about the device can be found in Sec. 6 .

We also tested 30 surfaces from the PhoTex database. ${ }^{29}$ This database consists of 3-D surface textures that were captured using a similar configuration with ours. Each surface was captured under different slant angles (30 deg, $45 \mathrm{deg}$, $60 \mathrm{deg}$, and $75 \mathrm{deg}$ ) and 12 tilt angles. We did not employ another dataset such as the one proposed by Shi et a ${ }^{16}$ because the objects in their dataset exhibit strong specular highlights and shadows, which are not in the scope of this paper.

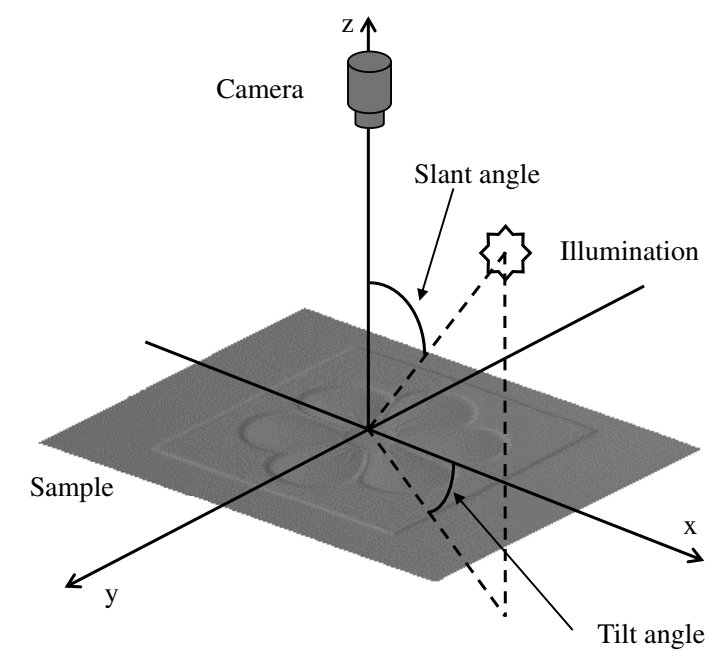

Fig. 3 The imaging setup. The illumination direction is defined by the slant angle and tilt angle as illustrated. 


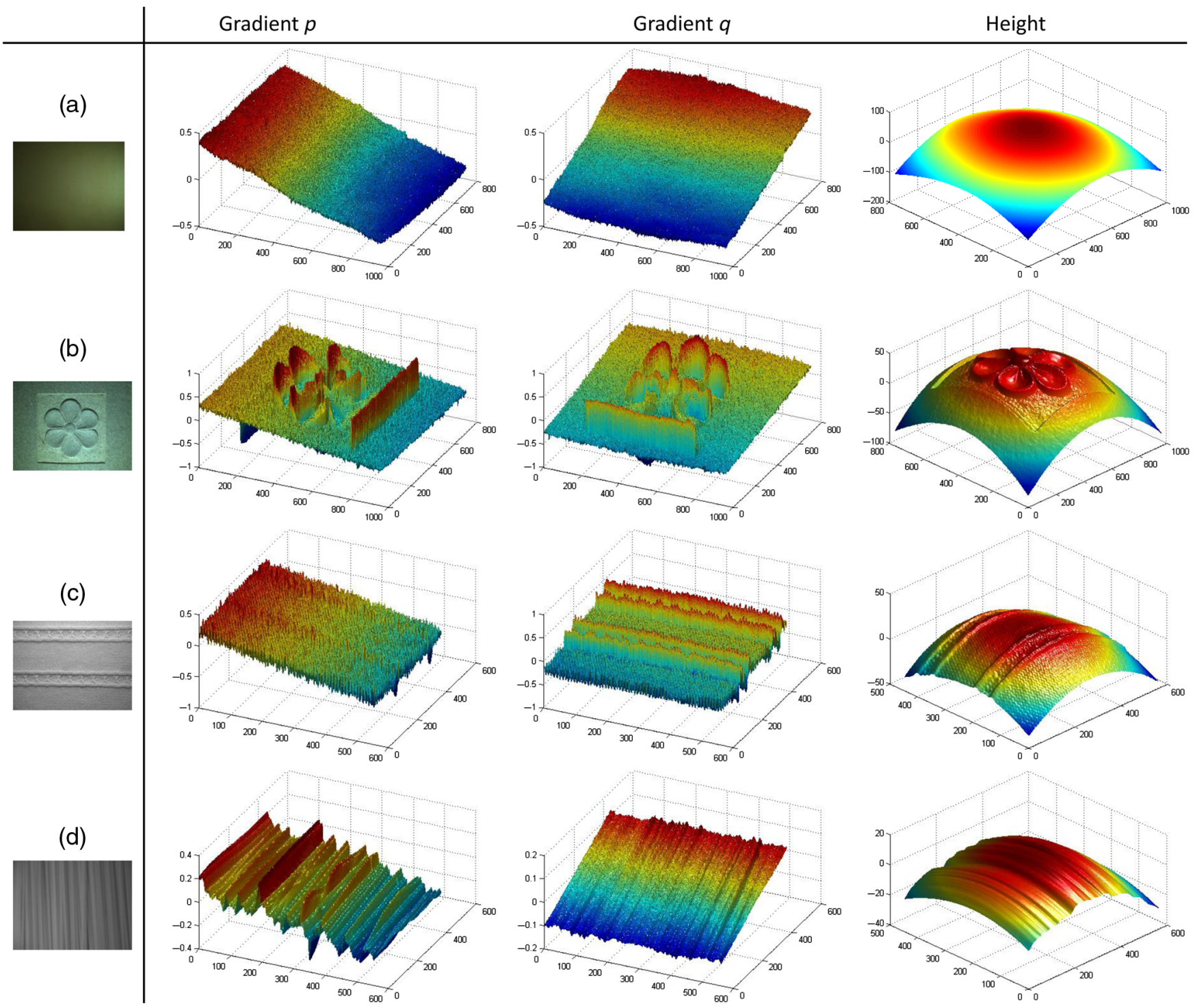

Fig. 4 Gradients and heights produced by classic photometric stereo: (a) a flat paperboard, (b) a flat flagstone, (c) surface texture "afa" in PhoTex database, and (d) surface texture "adj" in PhoTex database. Binary quadratic noise is very obvious in the height maps.

In this work, we recovered the gradients using classical photometric stereo and estimated the surface height using Poisson Solver. ${ }^{3}$ We plotted four surfaces in Fig. 4, from which it is obvious that the gradients show linear deviation, and the surface shape is contaminated by quadratic low-

Table 1 Statistics for fitting height maps with binary quadratic function.

\begin{tabular}{lcc} 
& $R^{2}$ & $p$ \\
\hline A flat paperboard & 0.9951 & $<0.001$ \\
A flat flagstone & 0.9867 & $<0.001$ \\
afa & 0.9917 & $<0.001$ \\
adj & 0.9674 & $<0.001$ \\
\hline
\end{tabular}

Note: "afa" and "adj" are from PhoTex database. frequency noise. Then we tested 36 samples (30 from PhoTex database, 6 self-captured) to validate the quadratic low-frequency deformation in recovered height maps by fitting a binary quadratic function. The $R^{2}$ statistic and $p$-value of the $F$ statistic are shown in Table 1 . All $R^{2}$ values are larger than 0.95 with $p<0.001$, which shows a significant quadratic relationship.

\subsection{Analysis for Gradient Deviation}

We denote the normal produced by photometric stereo in terms of gradients as $(-p,-q, 1)$. As shown in Fig. 5, $\mathbf{n}_{v}=$ $(0,0,1)$ denotes the vertical direction of a surface point, which is parallel to the optical axis, $\mathbf{n}_{g}=\left(-p_{g},-q_{g}, 1\right)$ is the "ground-truth" normal of the point, and $\mathbf{n}_{o}=\left(-p_{o}\right.$, $\left.-q_{o}, 1\right)$ is computed by the classic photometric stereo method with deviations. The $k$ 'th light direction is the principal direction $\mathbf{v}_{k}$. We denote the gradient deviation $p_{e}=p_{o}-p_{g}$ and $q_{e}=q_{o}-q_{g}$. The normal deviation $\mathbf{n}_{e}$ is 


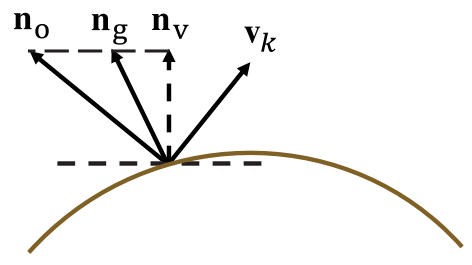

Fig. 5 The vertical direction $\mathbf{n}_{v}$, the "ground-truth" normal $\mathbf{n}_{g}$, the gradient normal estimated by the classic photometric stereo method $\mathbf{n}_{o}$, and the direction of the $k^{\prime}$ th light source $\mathbf{v}_{k}$.

$\mathbf{n}_{e}=\left(-p_{e},-q_{e}, 1\right)$.

The normal computed by classic photometric stereo can be corrected if $p_{e}$ and $q_{e}$ are estimated. The measured intensity $e_{k}$ can be redefined as

$$
\begin{aligned}
e_{k}= & \rho^{\prime}\left(-p_{o},-q_{o}, 1\right) \cdot \mathbf{v}_{k} \\
= & \rho^{\prime}\left(-p_{g},-q_{g}, 1\right) \cdot \mathbf{v}_{k}+\rho^{\prime}\left(-p_{e},-q_{e}, 1\right) \\
& \cdot \mathbf{v}_{k}-\rho^{\prime}(0,0,1) \cdot \mathbf{v}_{k}, \\
\rho^{\prime}\left(-p_{g},-q_{g}, 1\right) \cdot \mathbf{v}_{k}=\rho^{\prime}\left(-p_{o},-q_{o}, 1\right) & \\
& \cdot \mathbf{v}_{k}-\rho^{\prime}\left(-p_{e},-q_{e}, 1\right) \cdot \mathbf{v}_{k}+\rho^{\prime}(0,0,1) \cdot \mathbf{v}_{k},
\end{aligned}
$$

where $\rho^{\prime}=\rho /|\mathbf{n}|$.

When $p_{g}=0, q_{g}=0$ in Eq. (10), it means that the imaged surface is a flat plane. Then

$$
\begin{aligned}
\rho^{\prime}(0,0,1) \cdot \mathbf{v}_{k}= & \rho^{\prime}\left(-p_{o},-q_{o}, 1\right) \cdot \mathbf{v}_{k}-\rho^{\prime}\left(-p_{e},-q_{e}, 1\right) \\
& \cdot \mathbf{v}_{k}+\rho^{\prime}(0,0,1) \cdot \mathbf{v}_{k}
\end{aligned}
$$

In this situation, to satisfy the above equation for all light directions, we will have $p_{e}=p_{o}$ and $q_{e}=q_{o}$. This means that when we recover the shape of a flat plane under point light sources, the computed gradient is the gradient deviation.

If the surface height of the captured object is far smaller than the distance between the camera and the surface, the gradient estimated by a reference flat plane can be taken as the approximation of the gradient deviation. If we compute the gradient $\left(p_{e}, q_{e}\right)$ from a reference flat plane that has the same materials and colors, the corrected gradient $\left(p_{c}, q_{c}\right)$ will be

$\left(p_{c}, q_{c}\right)=\left(p_{o}-p_{e}, q_{o}-q_{e}\right)$.

However, providing various materials as reference flat planes is impractical. For a fixed photometric stereo setup, we can compute the gradients of a flat plane and use their distribution to optimize the estimated gradient of other surfaces.

\section{Gradient Correction Using Flat Plane Prior}

When photometric stereo is implemented under a fixed setup and the light sources bear the same attenuation, the recovered shapes of different surfaces share some deviation characteristics in common: (1) the low-frequency noise is approximately quadratic; (2) the centers of quadratic distributions can be considered to be the same position; (3) for different objects, the shape of the quadratic distributions is different. As gradients are the partial derivatives of the surface height, the gradient deviation shows a linear trend, and the slopes are different for different objects.

\subsection{Gradient Deviation Calibration}

From the previous experimental observations, we found that a binary quadratic function $f(x, y)=A x^{2}+B y^{2}+C x y+$ $D x+E y+F$ approximate the height deviation well. We propose to estimate the function parameters with a flat plane using classic photometric stereo and Poisson Solver. The gradient deviation and the quadratic center position will be

$$
\begin{aligned}
& p_{e}(x, y)=2 A x+C y+D, \quad q_{e}(x, y)=2 B y+C x+E, \\
& \left(x_{c}, y_{c}\right)=\left(\frac{C E-2 B D}{4 A B-C^{2}}, \frac{C D-2 A E}{4 A B-C^{2}}\right) .
\end{aligned}
$$

The deviation from a flat plane cannot satisfy all objects. We use it as optimization prior in the following solutions.

\subsection{Self-Correction Method}

Instead of using a flat plane, the object itself can be directly used to fit the gradient deviation distribution if it is globally flat (such as submarine topography).

We first compute the gradient and surface height by classic photometric stereo and Poisson Solver. A binary quadratic function $f(x, y)$ is used to fit the estimated surface height. The gradient deviation $\left(p_{e}, q_{e}\right)$ is derived from the fitted height and is subtracted from the original gradient $\left(p_{o}, q_{o}\right)$. The corrected gradient $\left(p_{c}, q_{c}\right)$, which is $\left(p_{o}-p_{e}\right.$, $\left.q_{o}-q_{e}\right)$, is finally used to integrate surface height.

However, object surfaces are not always planar, and this approach cannot provide the best result. To solve the problem, we further combine some points with known 3-D information to improve the accuracy.

\section{Optimization Algorithm for Gradient Deviation Correction}

If some surface points with known 3-D information are available, they can be used as constraints to improve the reconstruction accuracy. The gradient deviation will be corrected by minimizing the difference between the height produced by our methods and the known height of these points. The 3-D information of some surface points can be obtained by methods such as laser triangulation.

One problem to be noticed is that the result produced by photometric stereo is in pixel size, whereas the 3-D information produced by laser triangulation is in real size. Therefore, we have to first transform the photometric stereo result to real size.

\subsection{Scale Transformation of Photometric Stereo}

To implement the transformation between pixel and real scale, some known points are needed. In this paper, we make it by using laser triangulation (Fig. 6). A laser point $P\left(X_{p}, Y_{p}, Z_{p}\right)$ defined in camera coordinate system maps onto image point $\left(u_{p}, v_{p}\right)$. Then, the conversion factor can be written as

$\tau \approx\left|\frac{X_{p}}{u_{0}-u_{p}}\right|$, 

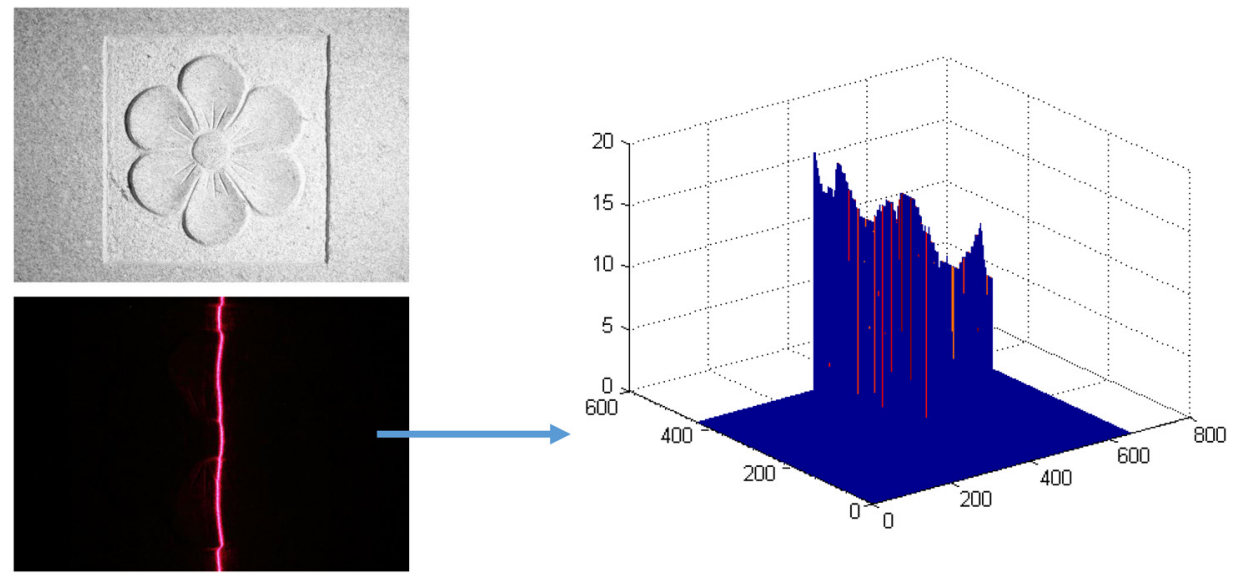

Fig. 6 Points with known 3-D information from laser triangulation.

where $u_{0}$ is the horizontal center of the physical image coordinate system, which can be calibrated by using Zhang's method. ${ }^{30}$

The object height produced by photometric stereo is defined as $H_{o}$ in pixel scale. The height of laser point $P$ estimated by photometric stereo is $h_{p}$ in pixel scale. Then, the real height $H_{p s}$ defined in the camera coordinate system can be transformed as

$$
H_{p s}=\left(Z_{p}+\tau \cdot h_{p}\right)-\tau \cdot H_{o}
$$

The real object height $H_{p s}$ corresponds to the $z$ coordinate in the camera coordinate system.

\subsection{Deviation Correction with Known Points as Priors}

The surface height from photometric stereo is transformed to real size first using the scale transformation method above. Then, we minimize the difference between photometric stereo result and the known height (from laser triangulation) to find the optimal parameters for the gradient deviation functions. The optimization equation is

$$
\begin{gathered}
\varepsilon=\min _{A, B, C, D, E}\left(\left|H_{l}-H_{p s}\left(p_{c}, q_{c}\right)\right|\right), \\
\quad \text { subject to } 2 A x_{0}+C y_{0}+D=0, \\
2 B y_{0}+C x_{0}+E=0
\end{gathered}
$$

where $H_{l}$ denotes the known height from laser triangulation and $H_{p s}\left(p_{c}, q_{c}\right)$ denotes the height produced by photometric stereo method in real size, where $p_{c}(x, y)=p_{0}(x, y)-$ $(2 A x+C y+D)$ and $q_{c}(x, y)=q_{0}(x, y)-(2 B y+C x+E)$.
With the best-fitted parameters of the gradient deviation functions, we can obtain the final surface height $H_{p s}$.

We initialize the parameters by using the results from the method described in Sec. 4. When the object is globally flat and parallel with the image plane, the result of the selfcorrection method would be preferred.

Let $\left(a_{0}, b_{0}, c_{0}, d_{0}, e_{0}\right)$ be the initial values for the parameters of the fitting function $f(x, y)$. The center of the quadratic function $\left(x_{0}, y_{0}\right)$ is assumed to be fixed and initialized by setting $p(x, y)=0$ and $q(x, y)=0$

$\left(x_{0}, y_{0}\right)=\left(\frac{c_{0} e_{0}-2 b_{0} d_{0}}{4 a_{0} b_{0}-c_{0}^{2}}, \frac{c_{0} d_{0}-2 a_{0} e_{0}}{4 a_{0} b_{0}-c_{0}^{2}}\right)$.

When we used the binary quadratic function $f(x, y)$ to fit the low-frequency deformation, we found that the value of parameter $C$ is much smaller than $A$ and $B$ (see Table 2 for the objects in Fig. 4). This means that the effect of parameter $C$ is trivial compared with other parameters. We explain this by the isotropic reflectance model photometric stereo assumes and proposed an optimization algorithm (Algorithm 1) to find the best parameters. We correct the gradient by adjusting parameters $(A, D)$ and $(B, E)$ simultaneously and take the parameter $C$ as constant. Figure 7 shows the corrected gradients and normals by our proposed method.

\section{Experiments}

\subsection{Experimental Setup}

\begin{tabular}{|c|c|c|c|c|c|c|}
\hline & para- $A$ & para- $B$ & para- $C$ & para- $D$ & para- $E$ & para- $F$ \\
\hline$a$ & $-3.94 \times 10^{-4}$ & $-4.49 \times 10^{-4}$ & $-2.98 \times 10^{-5}$ & 0.40 & 0.36 & -117.97 \\
\hline$b$ & $-2.76 \times 10^{-4}$ & $-3.50 \times 10^{-4}$ & $-2.32 \times 10^{-5}$ & 0.27 & 0.28 & -77.62 \\
\hline c & $-4.41 \times 10^{-4}$ & $-4.69 \times 10^{-4}$ & $1.42 \times 10^{-5}$ & 0.23 & 0.20 & -33.62 \\
\hline$d$ & $-2.59 \times 10^{-4}$ & $-2.48 \times 10^{-4}$ & $-5.55 \times 10^{-6}$ & 0.13 & 0.12 & -19.25 \\
\hline
\end{tabular}

Our experimental setup is shown in Fig. 8, including the photometric stereo system and the laser triangulation system.

Table 2 Statistics of fitting function.

Note: a: a flat paperboard, b: a flat flagstone, c: "afa" in PhoTex database, and d: "adj" in PhoTex database. 
Algorithm 1 Gradient-correction optimization method.

Input: Known sparse height: $H_{l}$, initial gradients $\left(p_{0}, q_{0}\right)$,

Initial coefficients $\left(a_{0}, b_{0}, c_{0}, d_{0}, e_{0}\right)$ and $\left(x_{0}, y_{0}\right)$.

1: $A=a_{0}, B=b_{0}, D=d_{0}, E=e_{0}$, para $=0.01$;

$Y_{1}=\operatorname{mean}\left(\left|H_{l}-H_{p s}\left(p_{0}, q_{0}\right)\right|\right)$

2: While not converged do

3: $\hat{A}_{k}=a_{0} \times\left[1+(-1)^{k} \times(k-1) \times\right.$ para $]$,

$\hat{B}_{k}=b_{0} \times\left[1+(-1)^{k} \times(k-1) \times\right.$ para $], \hat{D}_{k}=-\left(2 \hat{A}_{k} x_{0}+c_{0} y_{0}\right)$,

$\hat{E}_{k}=-\left(2 \hat{B}_{k} y_{0}+c_{0} x_{0}\right)$;

4: $p_{k}=p_{0}-\left(2 \hat{A}_{k} x+c_{0} y+\hat{D}_{k}\right) ; q_{k}=q_{0}-\left(2 \hat{B}_{k} y+c_{0} x+\hat{E}_{k}\right)$;

5: $T=\operatorname{mean}\left(\left|H_{l}-H_{p s}\left(p_{k}, q_{k}\right)\right|\right)$;

6: if $T<Y_{k}$

7: $A=\hat{A}_{k}, B=\hat{B}_{k}, D=\hat{D}_{k}, E=\hat{E}_{k}, Y_{k}=T$;

8: end if

9: $Y_{k+1}=Y_{k}$;

10: end while

Output: $A, B, D, E$

A camera (IDS UI-358xCP-C) and six light sources constituted the photometric stereo system. The camera was placed at the center of the system, surrounded by six light-emitting diode (LED) point light sources. The light sources with adjustable lighting direction were placed around a rotating circular orbit. The line-structured laser was placed alongside the camera. The laser and the camera constituted a line laser
3-D imaging system. The objects were placed below the camera.

In our experiments, the distance between the camera and objects along the optical axis was about $574 \mathrm{~mm}$. The slant angle of each light source was set to $45 \mathrm{deg}$, and the distance between the light sources and objects along the optical axis was about $375 \mathrm{~mm}$, which is similar to the radius of the circular orbit. The camera's resolution was $1280 \times 1024$ pixels, in which case each pixel is $\sim 0.273 \mathrm{~mm}$. The images were captured in a dark room, and the LED lights are the only light sources. Photometric stereo was computed under six light directions, which had the same interval along the circular orbit.

\subsection{Method Evaluation}

We compared our method with the laser triangulation, the result of which was regarded as ground truth. In our experiments, two perpendicular laser lines were employed in laser triangulation. $25 \%$ of the laser points were randomly selected and served as constraints in the optimization step of our method, and they were not integrated to final results. Therefore, the laser points could be used as ground truth for error analysis.

We compared our method with Woodham's ${ }^{1}$ and Agrawal's ${ }^{3}$ work (classic PS), which directly recovered the object's surface. We also compared it with Angelopoulou and Petrou's ${ }^{10}$ work (flat fielding), which calibrated the light distribution in advance. We analyzed errors of a flat paperboard, a flat flagstone, and a flat slab with embedded shells. The height maps produced by photometric stereo were transformed from pixel size to real size, and the average error was estimated in real size. The error analysis is shown in Table 3. The section profiles of the three objects on one projected laser line are shown in Figs. 9, 10, and 11. The reconstruction figures are shown in Fig. 12.

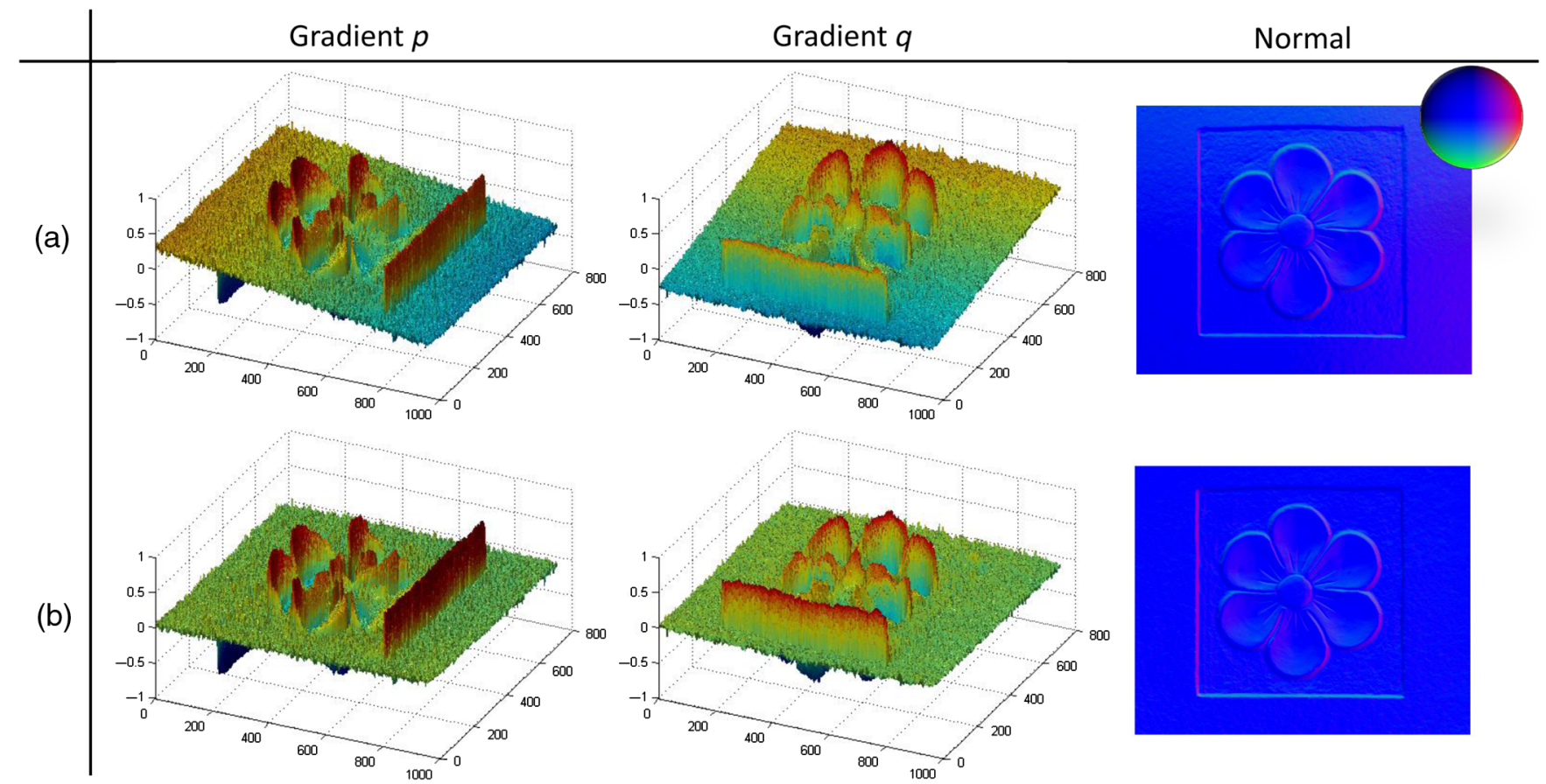

Fig. 7 Gradients and normal corrected by our optimization method: (a) the original gradients and normal produced by classic photometric stereo and (b) the corrected results by our method. 

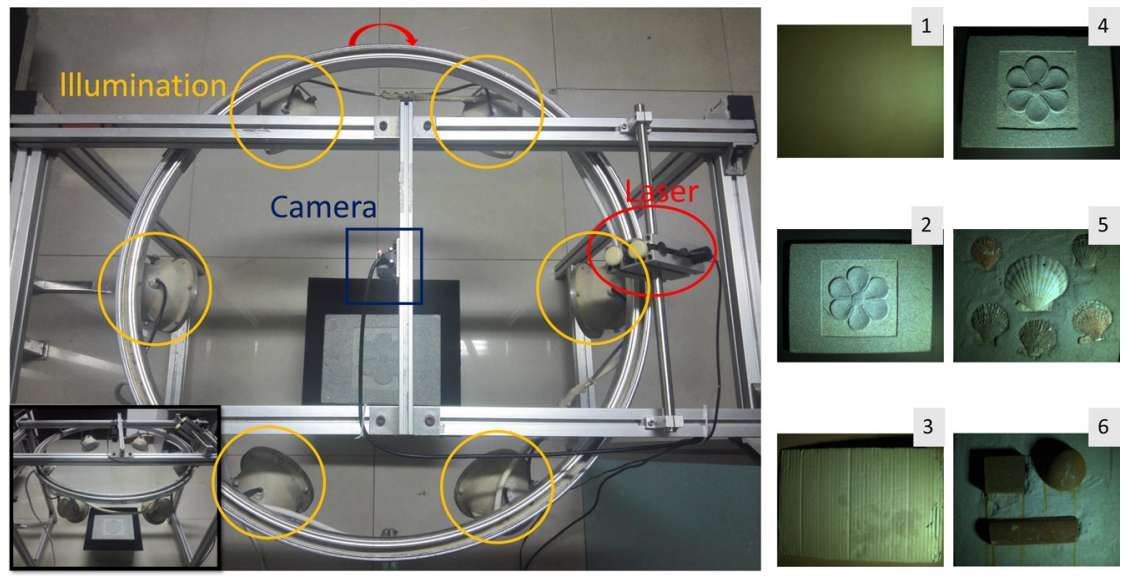

Fig. 8 The experimental setup and the six target objects: (1) a flat paperboard, (2) a flat flagstone, (3) a tilted paperboard, (4) a tilted flagstone, (5) a slab with shells, and (6) a slab with steel sheets.

Table 3 Method comparison.

\begin{tabular}{lccc}
\hline Error $(\mathrm{mm})$ & $\begin{array}{c}\text { A flat } \\
\text { paperboard }\end{array}$ & $\begin{array}{c}\text { A flat } \\
\text { flagstone }\end{array}$ & Shells \\
\hline${\text { Classic } \mathrm{PS}^{1}}^{10}$ & 7.7610 & 5.6282 & 6.9351 \\
Flat fielding $^{10}$ & 1.6276 & 1.0748 & 2.0853 \\
OGSC & $\mathbf{0 . 3 9 5 8}$ & $\mathbf{0 . 4 3 0 8}$ & $\mathbf{0 . 9 2 6 9}$ \\
\hline
\end{tabular}

Note: Bold values represent that the results are best and the related methods are better than others.

Among the methods, our approach shows smaller errors and is closer to the ground truth (results from laser triangulation). The left column of Fig. 12 is the result produced by classic photometric stereo, from which the deformed surface shape is obviously seen. The right column of Fig. 12 exhibits the corrected results by our proposed method. Figure 13 shows the corrected normals and 3-D shapes. The low-frequency noise is well-eliminated with high-frequency details wellreserved (the crack on the shell is clearly visible). Our OGSC method shows better performance for globally flat objects.

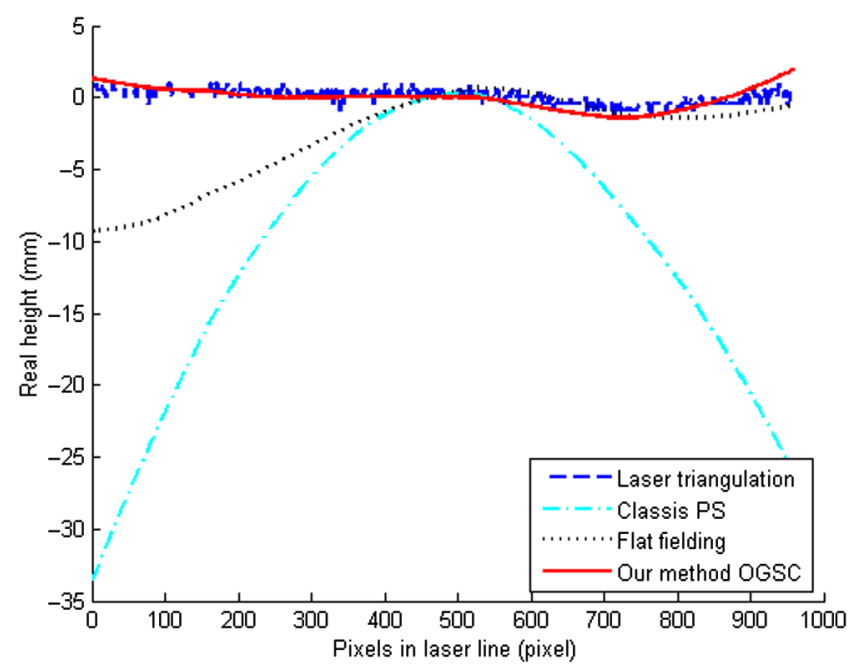

Fig. 9 Section profiles of the height maps for a flat paperboard.

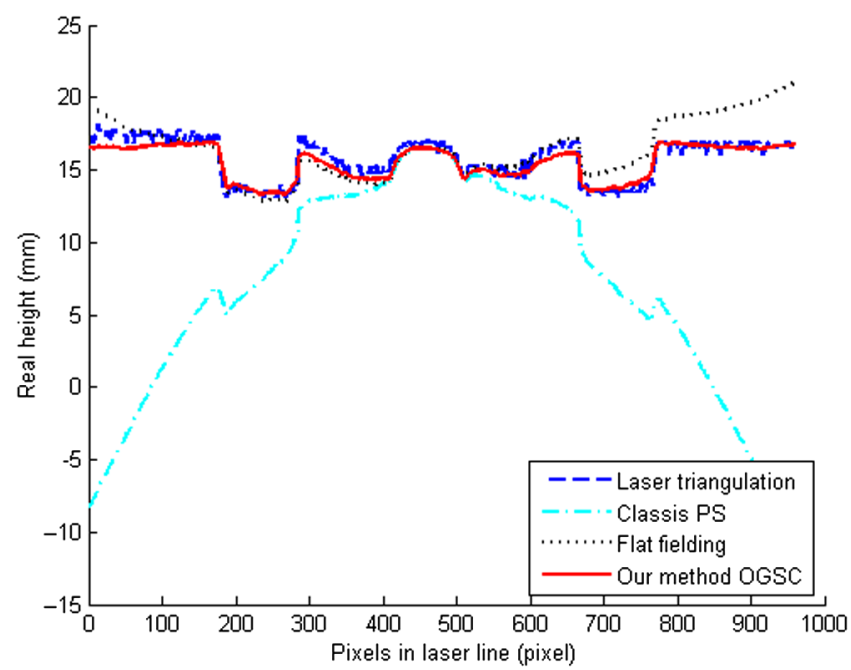

Fig. 10 Section profiles of the height maps for a flat flagstone.

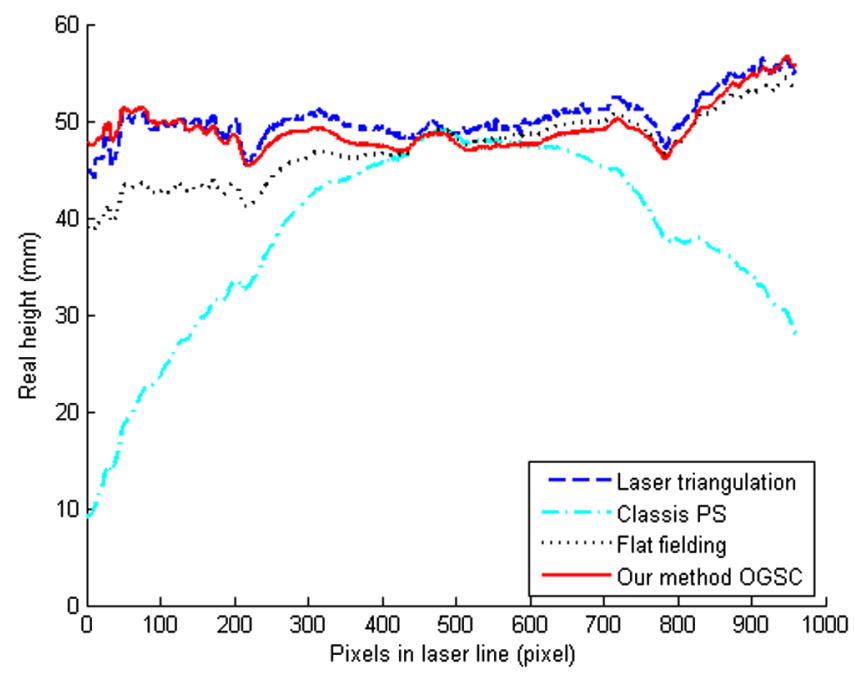

Fig. 11 Section profiles of the height maps for a slab with embedded shells. 


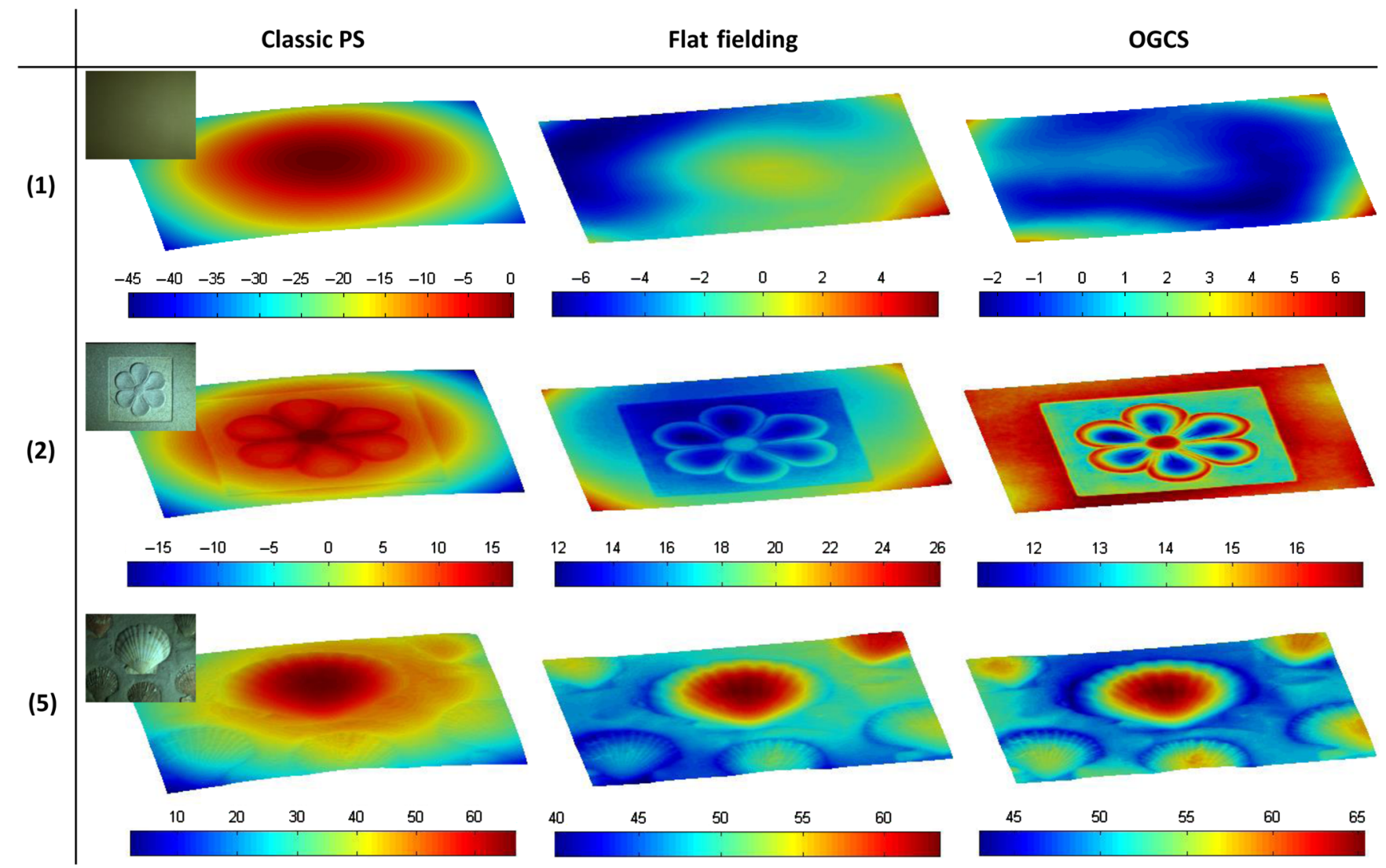

Fig. 12 Comparison of different methods on samples (1), (2), and (5). The color of the reconstruction mesh codes the height.

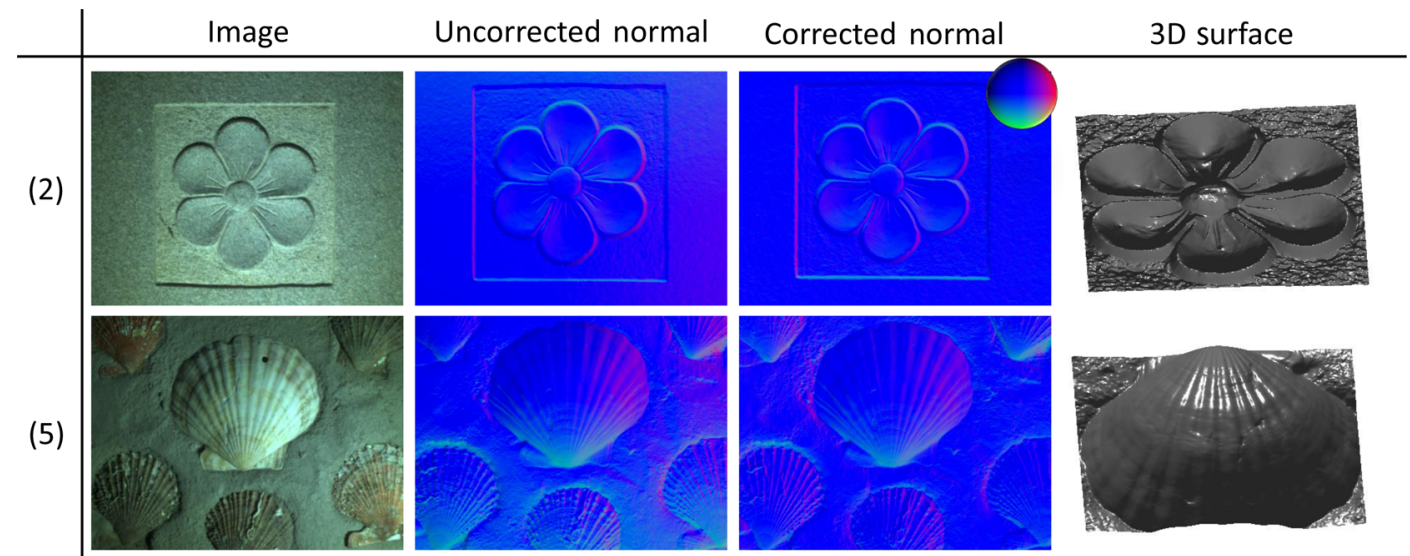

Fig. 13 The corrected normal maps and rendered images of the reconstruction results from our OGSC method.

\subsection{Quantitative Evaluation}

To quantitatively evaluate our method, we tested different types of objects, including flat objects, tilted objects, and convex objects. The results are shown in Fig. 14.

From the error analysis (Table 4) on flat objects (samples 1 and 2), it can be seen that the precision of our methods is below $1 \mathrm{~mm}$. The quantitative results from the table and Fig. 14 indicate that the OGCS method performs better than other methods for the globally flat objects.
We further tested our methods on tilted objects and convex objects. Table 5 shows the results of a tilted paperboard and a tilted flagstone. The OGC method produced better performances, whereas the other methods all failed. The convex objects include a slab with embedded shells and a slab with steel sheets. From Table 6, it can be seen that our methods are proved to work well on continuous surface but cannot handle noncontinuous surface, which is outside the scope of photometric stereo. The embedded shells with continuous surface 


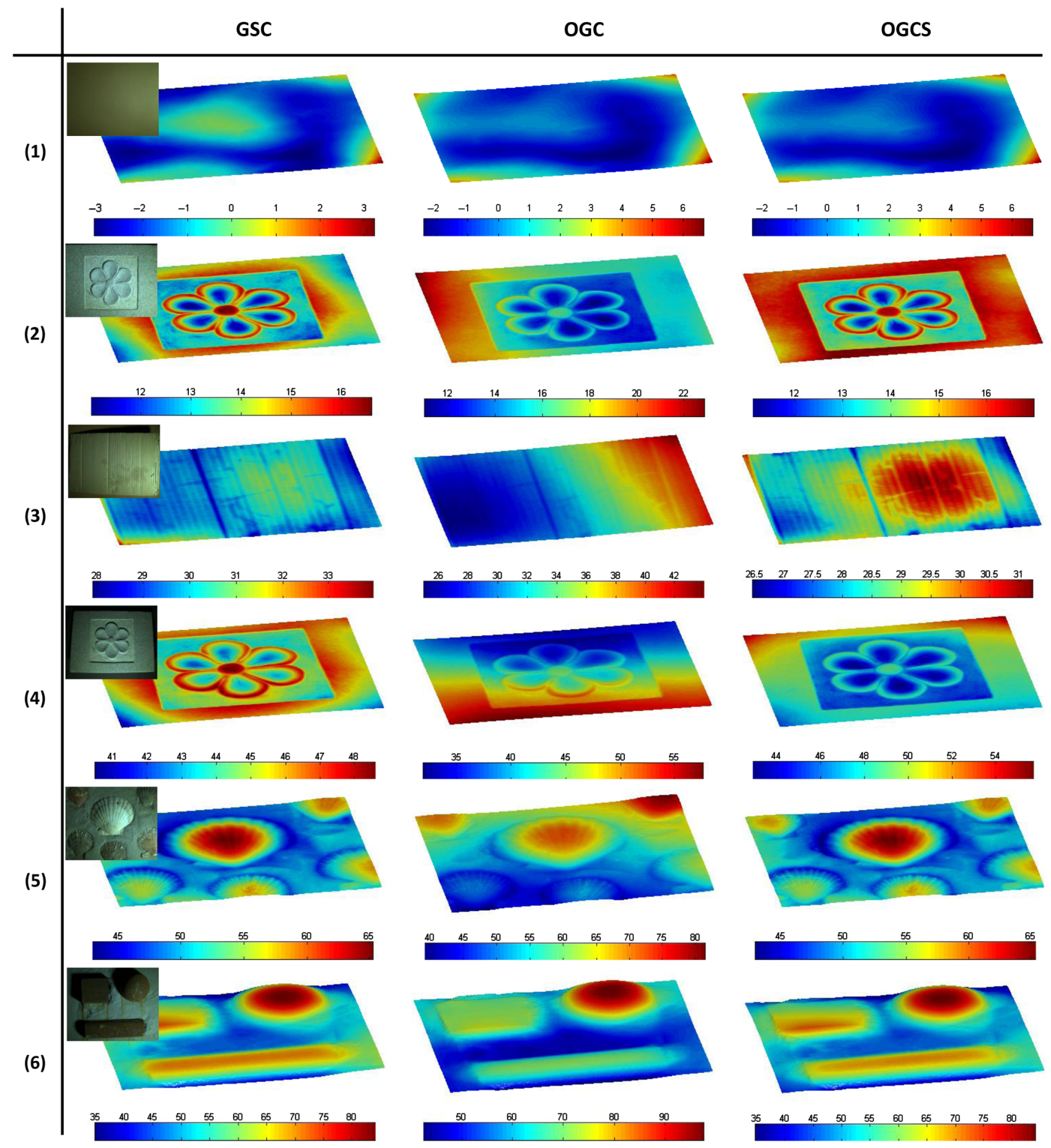

Fig. 14 Reconstruction results for sample objects by our methods.

were reconstructed well, but the steel sheets with noncontinuous surface performed poorly and produced larger errors.

The OGSC method initialized from the object itself provides better performance on globally flat objects, whereas the OGC method using the calibrated initialization can handle more situations.

In close-range photometric stereo, the attenuation of lighting becomes worse when moving from the imaging center to the surround. We, therefore, tested the performance decay of our proposed method with increasing imaging field. As shown in Fig. 15, the original images of two samples were cropped to three different subimages. If the size of the original image is $R$, the size of cropped images is $R / 16, R / 4$, and $9 R / 16$. The cropped images are located in the central regions of the original image. From the results shown in Fig. 16, we can see that the performance of classic photometric stereo drops dramatically when including larger imaging areas of the objects, whereas our approach exhibits steady 
Fan et al.: Deviation correction method for close-range photometric stereo...

Table 4 Error analysis of flat objects.

\begin{tabular}{lcc} 
Error $(\mathrm{mm})$ & A flat paperboard & A flat flagstone \\
\hline Classic PS $^{1}$ & 7.7610 & 5.6282 \\
GSC & 1.0466 & 0.8529 \\
OGC & 0.3958 & 0.8200 \\
OGSC & $\mathbf{0 . 3 9 5 8}$ & $\mathbf{0 . 4 3 0 8}$ \\
\hline
\end{tabular}

Note: Bold values represent that the results are best and the related methods are better than others.

Table 5 Error analysis of tilted objects.

\begin{tabular}{lcc} 
Error $(\mathrm{mm})$ & A tilted paperboard & A tilted flagstone \\
\hline Classic $\mathrm{PS}^{1}$ & 8.1613 & 8.1958 \\
GSC & 4.4365 & 6.2643 \\
OGC & $\mathbf{2 . 2 0 9 1}$ & $\mathbf{2 . 7 3 1 0}$ \\
OGSC & 4.335 & 6.1249 \\
\hline
\end{tabular}

Note: Bold values represent that the results are best and the related methods are better than others.

Table 6 Error analysis of convex objects.

\begin{tabular}{lcc}
\hline Error $(\mathrm{mm})$ & Shells & Steel sheets \\
\hline Classic PS & 6.9351 & 13.2174 \\
GSC & 0.9327 & 8.9068 \\
OGC & 2.6935 & 7.4100 \\
OGSC & $\mathbf{0 . 9 2 6 9}$ & 8.6280 \\
\hline
\end{tabular}

Note: Bold value represents that the results are best and the related methods are better than others.

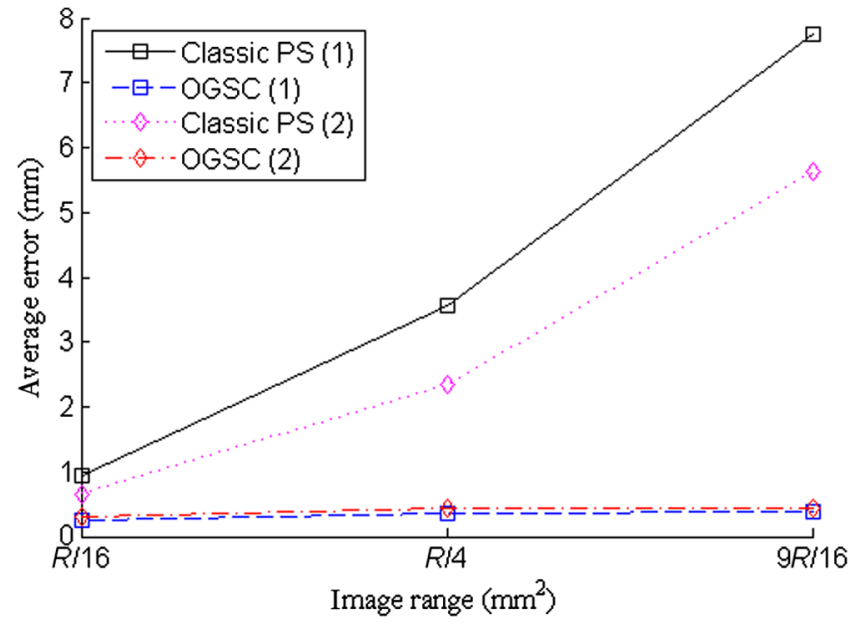

Fig. 16 Average error analysis about the imaging range: (1) a flat paperboard and (2) a flat flagstone.

reconstruction errors. This means that our approach improves photometric stereo in working on a larger imaging area and is therefore more useful for practical applications.

Table 7 The restriction of different light distribution.

\begin{tabular}{|c|c|c|c|c|c|}
\hline & Error (mm) & 3 lights & 4 lights & 6 lights & 12 lights \\
\hline \multirow[t]{2}{*}{1} & Classic PS ${ }^{1}$ & 7.7419 & 8.0078 & 7.7610 & 7.7582 \\
\hline & OGSC & 2.1671 & 2.5273 & 0.3958 & 0.4234 \\
\hline \multirow[t]{2}{*}{2} & Classic PS ${ }^{1}$ & 5.8758 & 6.3836 & 5.6282 & - \\
\hline & OGSC & 1.7761 & 2.1991 & 0.4308 & - \\
\hline
\end{tabular}

Note: Bold values represent that the results are best and the related methods are better than others.

Note: 1: a flat paperboard and 2: a flat flagstone.

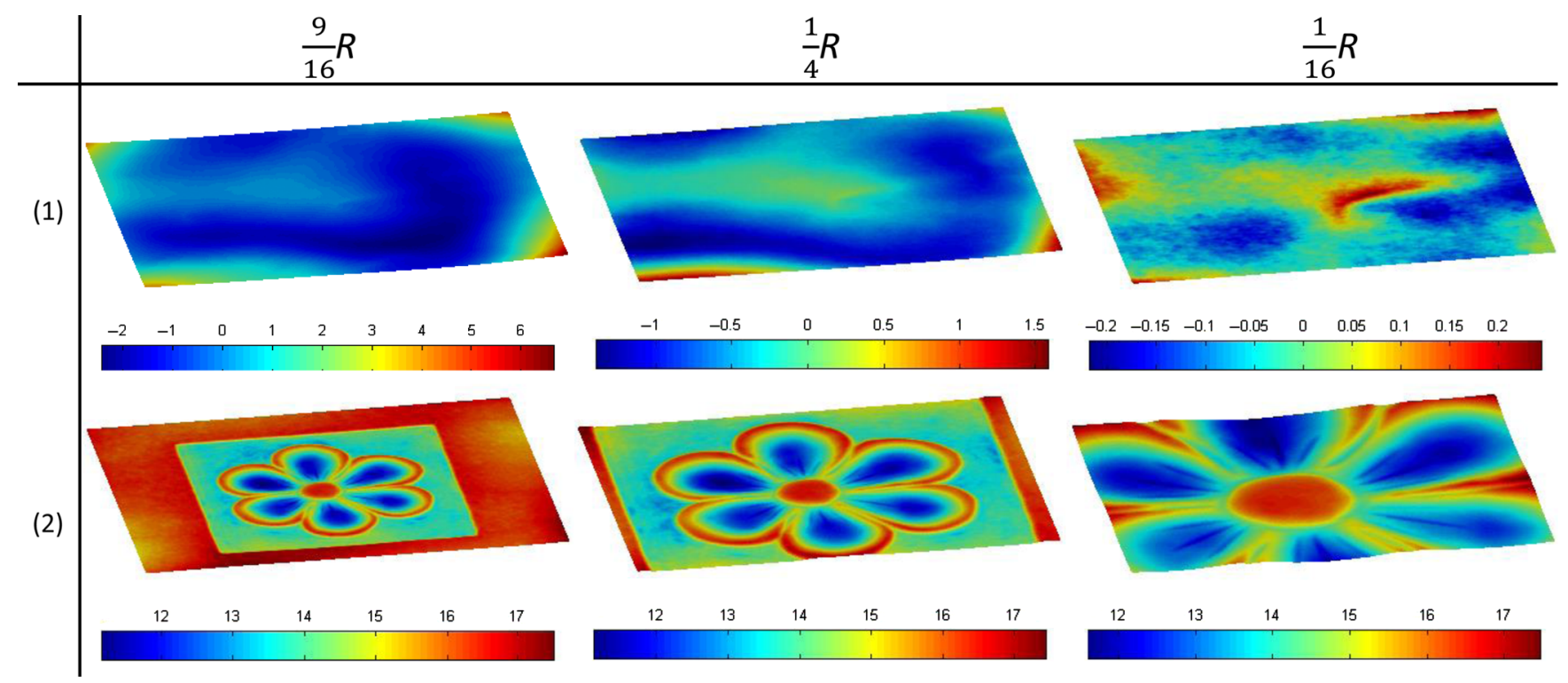

Fig. 15 Results of flat samples in different imaging range, which are central regions of the original image. $R$ means the size of the original image: $350 \mathrm{~mm} \times 280 \mathrm{~mm}$. (1) A flat paperboard and (2) a flat flagstone. 
We also tested our methods under a different combination of lighting directions, including symmetric and asymmetric lighting distribution. Twelve lighting directions with different tilt angles were used. The light sources used in each test are coded in the bottom right corner of Fig. 17. We tested a flat paperboard and a flat flagstone. The results in Table 7 and Fig. 17 show that the symmetric lighting distribution provides better performance in terms of details. This indicates that our method can better deal with images captured under symmetric lighting.

Finally, we tested the proposed method using the PhoTex database. Since the database does not provide ground truth or any points with known 3-D information, we used the proposed GSC method. We chose three samples, and for each

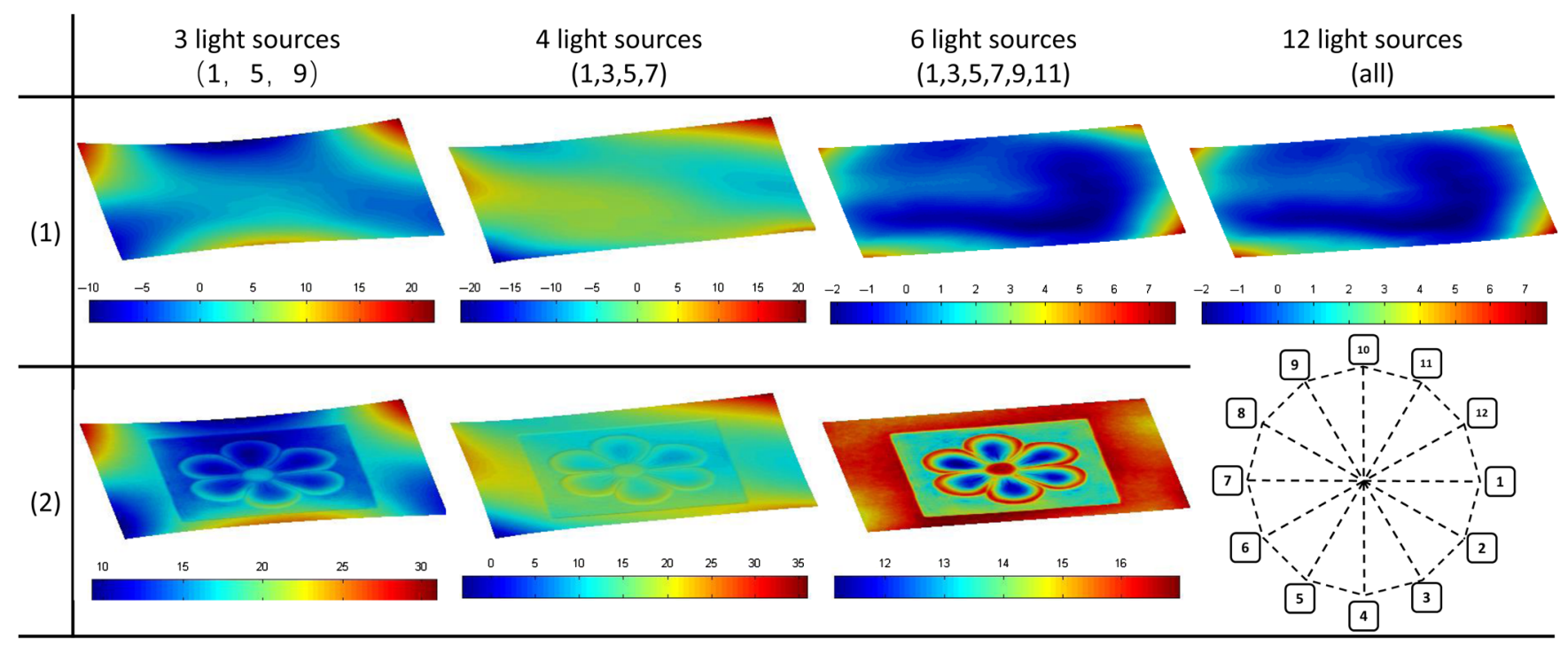

Fig. 17 Results under different light distributions, including asymmetric light distribution and symmetric light distribution. The light distribution is shown at the bottom right corner: (1) a flat paperboard and (2) a flat flagstone.

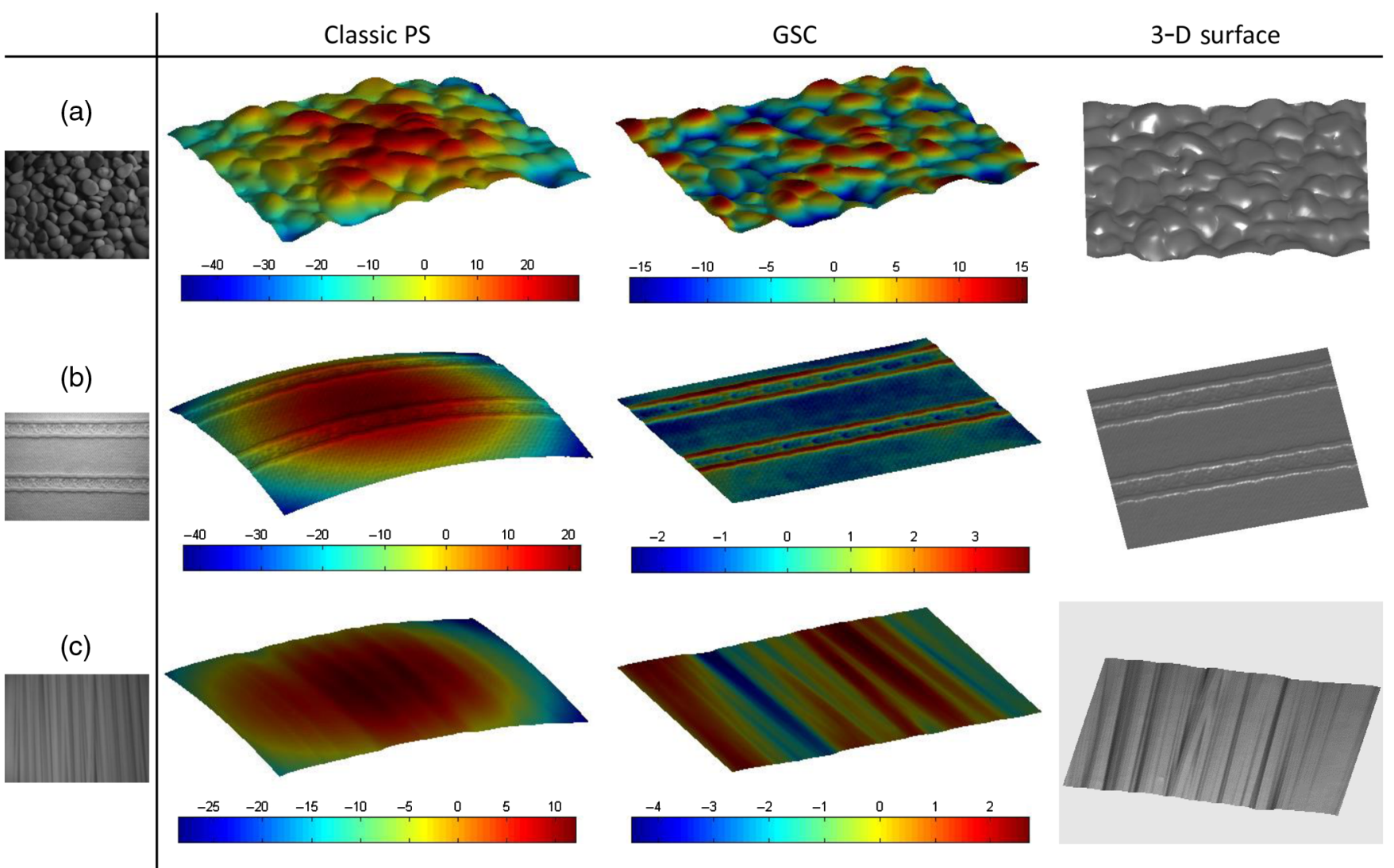

Fig. 18 Experiments of real objects from PhoTex database: (a) "ade" (stones), (b) "afa" (a piece of fabric), and (c) "adj" (noodles). 
sample, we chose 12 images with the same slant angle (45 deg). The results are shown in Fig. 18. The samples "ade" (stones) and "adj" (noodles) exhibit heavy shadows, but our method can still reduce the low-frequency noise and reserve surface details.

\section{Conclusion}

In this paper, we proposed methods to correct the deviation produced by classic photometric stereo under point light sources. Based on the observation that the deviation in gradients shows a linear trend, a GSC method is proposed for deviation correction. Optimization methods are further developed by combining some surface points with known 3-D information. Our method improves the photometric stereo in dealing with nonuniform illuminations, which will be useful for situations that the light sources cannot be placed far away from the camera and for large reconstruction scenes. Future work will include improving the accuracy of the approach and handling globally curved surfaces and moving objects.

\section{Acknowledgments}

This work was supported by the International Science and Technology Cooperation Program of China (No. 2014DFA10410) and the National Natural Science Foundation of China (No. 61501417).

\section{References}

1. R. J. Woodham, "Photometric method for determining surface orientation from multiple images," Opt. Eng. 19(1), 191139 (1980).

2. R. T. Frankot and R. Chellappa, "A method for enforcing integrability in shape from shading algorithms," IEEE Trans. Pattern Anal. Mach. Intell. 10(4), 439-451 (1988).

3. A. Agrawal, R. Raskar, and R. Chellappa, "What is the range of surface reconstructions from a gradient field?," in European Conf. on Computer Vision, pp. 578-591, Springer (2006)

4. M. Harker and P. O'Leary, "Regularized reconstruction of a surface from its measured gradient field," J. Math. Imaging Vision 51(1), 46-70 (2015).

5. S. D. Cochran and G. Medioni, "3-D surface description from binocular stereo," IEEE Trans. Pattern Anal. Mach. Intell. 14(10), 981-994 (1992).

6. S. Agarwal et al., "Building Rome in a day," Commun. ACM 54(10), 105-112 (2011).

7. J. Ackermann et al., "A survey of photometric stereo techniques," Found. and Trends ${ }^{\circledR}$ Comput. Graphics Vis. 9(3-4), 149-254 (2015).

8. Y. Furukawa et al., "Multi-view stereo: a tutorial," Found. Trends Comput. Graphics Vision 9(1-2), 1-148 (2015).

9. N. Kolagani, J. S. Fox, and D. R. Blidberg, "Photometric stereo using point light sources," in Proc. 1992 IEEE Int. Conf. on Robotics and Automation, pp. 1759-1764, IEEE (1992).

10. M. E. Angelopoulou and M. Petrou, "Evaluating the effect of diffuse light on photometric stereo reconstruction," Mach. Vision Appl. 25(1), 199-210 (2014).

11. S. Li and B. Shi, "Photometric stereo for general isotropic reflectances by spherical linear interpolation," Opt. Eng. 54(8), 083104 (2015).

12. H. Fan et al., "Robust photometric stereo in a scattering medium via low-rank matrix completion and recovery," in 9th Int. Conf. on Human System Interactions (HSI), pp. 323-329, IEEE (2016).

13. T. Collins and A. Bartoli, "3D reconstruction in laparoscopy with closerange photometric stereo," in Int. Conf. on Medical Image Computing and Computer-Assisted Intervention, pp. 634-642, Springer (2012).

14. I. Horovitz and N. Kiryati, "Depth from gradient fields and control points: bias correction in photometric stereo," Image Vision Comput. 22(9), 681-694 (2004).

15. D. Cho et al., "Photometric stereo under non-uniform light intensities and exposures," in European Conf. on Computer Vision, pp. 170-186, Springer (2016)

16. B. Shi et al., "A benchmark dataset and evaluation for non-Lambertian and uncalibrated photometric stereo," in Proc. of the IEEE Conf. on Computer Vision and Pattern Recognition, pp. 3707-3716 (2016).

17. A. Tankus and N. Kiryati, "Photometric stereo under perspective projection," in Tenth IEEE Int. Conf. on Computer Vision (ICCV'05), Vol. 1, pp. 611-616, IEEE (2005).
18. T. Higo et al., "A hand-held photometric stereo camera for 3-D modeling," in IEEE 12th Int. Conf. on Computer Vision, pp. 1234-1241, IEEE (2009).

19. J. Wang et al., "Photometric stereo with small angular variations," in Proc. of the IEEE Int. Conf. on Computer Vision, pp. 3478-3486 (2015).

20. A. Wetzler et al., "Close-range photometric stereo with point light sources," in 2nd Int. Conf. on 3D Vision, Vol. 1, pp. 115-122, IEEE (2014).

21. F. Logothetis et al., "Near-field photometric stereo in ambient light," in British Machine Vision Conference (2016).

22. F. Logothetis, R. Mecca, and R. Cipolla, "Semi-calibrated near field photometric stereo," in Computer Vision and Pattern Recognition (CVPR) (2017).

23. Y. Quéau, T. Wu, and D. Cremers, "Semi-calibrated near-light photometric stereo," in Int. Conf. on Scale Space and Variational Methods in Computer Vision (SSVM) (2017).

24. Y. QUeau et al., "A non-convex variational approach to photometric stereo under inaccurate lighting," in Proc. Computer Vision and Pattern Recognition (CVPR) (2017).

25. D. Nehab et al., "Efficiently combining positions and normals for precise 3D geometry," ACM Trans. Graphics 24(3), 536-543 (2005).

26. M. Haque et al., "High quality photometric reconstruction using a depth camera," in Proc. of the IEEE Conf. on Computer Vision and Pattern Recognition, pp. 2275-2282 (2014).

27. J. Dong et al., "Improving photometric stereo with laser sectioning," in IEEE 12th Int. Conf. on Computer Vision Workshops (ICCV Workshops), pp. 1748-1754, IEEE (2009).

28. J. Dong, "Three-dimensional surface texture synthesis," $\mathrm{PhD}$ Thesis, Heriot-Watt University (2003).

29. Texturelab Edinburgh, "PhoTex: a texture database," http://www.macs. hw.ac.uk/texturelab/resources/databases/photex/ (2013).

30. Z. Zhang, "A flexible new technique for camera calibration," IEEE Trans. Pattern Anal. Mach. Intell. 22(11), 1330-1334 (2000).

Hao Fan received his BSc and MSc degrees from the Department of Computer Science and Technology, Ocean University of China, in 2012 and 2014, respectively. Currently, he is a PhD candidate of computer application technology in the Department of Computer Science and Technology, Ocean University of China, Qingdao, China. His research interests include computer vision, three-dimensional reconstruction, and underwater image processing.

Lin Qi received his BSc and MSc degrees from the Ocean University of China in 2005 and 2008, respectively, and received his PhD in computer science from Heriot-Watt University in 2012. He is now working in the Department of Computer Science and Technology, Ocean University of China. His research interests include computer vision and visual perception.

Nan Wang is a MS student in Vision Lab of Ocean University of China. He received his BSc degree in computer science and technology from Ocean University of China in 2014. His research interest includes $3 \mathrm{D}$ reconstruction and underwater image processing.

Junyu Dong received his BSc and MSc degrees from the Department of Applied Mathematics, Ocean University of China in 1993 and 1999, respectively. He received his $\mathrm{PhD}$ in image processing from the Department of Computer Science, Heriot-Watt University, UK, in November 2003. He joined the Ocean University of China in 2004, and he is currently a professor and the head of the Department of Computer Science and Technology. His research interest includes machine learning, big data, computer vision, and underwater image processing.

Yijun Chen received his BS degree in mechatronic engineering from Northeast Forestry University, Harbin, China. Currently, he is a graduate student in computer technology in the Department of Computer Science and Technology, Ocean University of China, Qingdao, China. His research interests include machine learning, image processing and computer vision.

Hui Yu received his $\mathrm{PhD}$ in graphics from Brunel University, UK. He is a reader with the University of Portsmouth, UK. His research interests include vision, computer graphics and application of machine learning to above areas, particularly in image/video processing and recognition, human computer interaction, human behavior understanding, robotics, 3-D reconstruction, and geometric processing of human/ facial performances. He is an associate editor of IEEE Transactions on Human-Machine Systems. 